\title{
Compensation for the demodulated phase errors caused by sampling frequency offset in OFDM systems
}

\author{
Yujie Xiong, Xiaofei Zhang, Yi Zhang \\ School of Information and Electronic \\ Beijing Institute of Technology, BIT \\ Beijing, P.R China \\ pure_tide@163.com, xiaofeizhangsun@gmail.com, daviddy1955@sina.com
}

\begin{abstract}
OFDM has been successfully adopted in Digital video broadcasting-terrestrial (DVB-T) due to its excellent spectral efficiency and inherent resistance to multipath effect. However, OFDM is a multi-carrier transmission technique and sampling frequency offset (SFO) always exists in OFDM systems. It must be noticed that SFO would result in interference between adjacent sub-carriers (ICI) as well as demodulated phase deviation and seriously degrade performance of demodulation. Therefore, in this paper we propose an adaptive sampling frequency tracking and demodulated phase error compensation scheme based on synchronization symbol information of OFDM signals. We achieved correction of phase errors and solved the problem that OFDM signals cannot be correctly demodulated for complex constellation when SFO exists. Infield test results of the proposed scheme in an ADSL environment indicate great performance improvement of demodulation. type1.
\end{abstract}

Keywords-OFDM; sampling frequency Offset; phase deviation.

\section{INTRODUCTION}

Due to its excellent spectral efficiency and inherent resistance to multipath effect, OFDM is very suitable for high-data-rate and multi- media transmission. Commercial OFDM based systems include Digital Audio Broadcasting (DVB), Digital Video Broadcasting (DVB), Wireless Local Area Networks (WLAN), and Asymmetric Digital Subscriber Line (ADSL), etc[1]-[3]. In addition, OFDM is also regarded as a competitive candidate for the physical technology of future mobile cellular systems. In a classic OFDM transmission scenario, a symbol which comprises several orthogonal subcarriers modulated by independent data streams are generated by an IFFT procedure at the transmitter, passing through the channel, and then demodulated by a corresponding FFT procedure at the receiver [4]. During this process, the orthogonality between the subcarriers must be guaranteed, as it is critically important for the synchronization and demodulation of the received signal.

For wireless communication systems where the transmitter and the receiver do not share a common clock source, there will always be a frequency offset between their

\footnotetext{
This work was supported in part by the National Natural Science Foundation of China (Grant NO.61072050)

All the authors are with the Department of Information and Electronics, Beijing Institute of Technology, Beijing 100081, P. R. China (email:xiaofeizhangsun@gmail.com)
}

sampling clocks. Moreover, both of these sampling clocks are inevitably subject to random jitters and they jitter uncorrelatedly. Such non-coherence between the sampling clocks at the transmitter and receiver may destroy the orthogonality among the subcarriers within the OFDM symbols, and thereby leads to imperfect mitigation of ICI and a phase deviation at the demodulator's output, which in turn make correct detection of the received signal very difficult or even impossible. In most existing literatures on OFDM receiver, sampling clock is either assumed ideal (frequency \& phase coherent with that of the transmitter) or to have a very small offset $\xi$ satisfying $\xi+\mathrm{T}<\mathrm{L}$, where $\mathrm{T}$ and $\mathrm{L}$ stand for the maximum delay spread and the length of cyclic prefix, respectively. Given the " $\xi+\mathrm{T}<\mathrm{L}$ " assumption, the effect of sampling clock offset on the orthogonality of the subcarriers is typically weak enough to be overlooked for a small or medium order of constellation (e.g. $\leq 64$ ), and the demodulation error, which now reflects the phase deviation only, can also be kept at a satisfactorily low level to meet the basic requirements of symbol detection. However, for more complicated constellation (e.g. $\geq 64$ ), the performance of demodulator will be degraded unacceptably because of the phase noise, even the above mentioned " $\xi+\mathrm{T}<\mathrm{L}$ " assumption holds [5].

In this paper we propose an adaptive sampling frequency tracking and demodulated phase error compensation scheme. In-field test results of the proposed scheme in an ADSL environment are also presented, which indicate great performance improvement of demodulator.

\section{ANALYSIS OF ADAPTIVE SAMPLING FREQUENCY TRACKING \& DEMODULATED PHASE DEVIATION COMPENSATION SCHEME BASED ON SYNCHRONIZED SYMBOL INFORMATION}

\section{A. Analysis of the correlation between SFO and demodulated phase}

As a result of the mismatch between the sampling clocks at the transmitter and the receiver, each sample of the received signal will be deviated a little bit from its punctual timing position. Understandably, such deviation increases linearly with the amount of samples, and leads to phase rotation in each of the OFDM subcarriers. Moreover, even the SFO is correctly compensated; the initial phase offset shall still result in a demodulated phase deviation. In Fig.1 we plot the complex demodulated output of three consecutive symbols for two different subcarriers with adjacent center 
frequencies in rectangular coordinate system. It can be seen in the figure that the SFO (SFO) affect the demodulated phase in two ways. On one hand, the phase rotation caused by SFO on a certain subcarrier increase linearly with the symbol indices; on the other hand, if we define the phase deviation on $\mathrm{k}$-th subcarrier as $\Delta \theta_{k}$, then for any $\mathrm{k} 1>\mathrm{k} 2$, we shall have $\Delta \theta_{k 1}>\Delta \theta_{\mathrm{k} 2}$. i.e., subcarriers with higher center frequency will always suffer more phase deviation, as indicated by the figure.

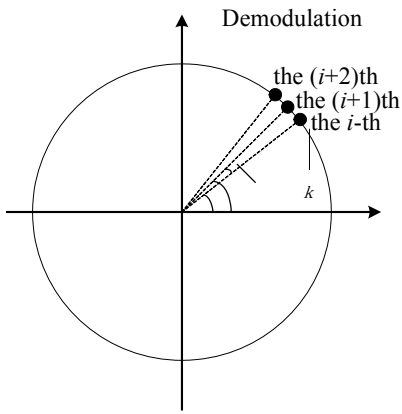

the $k$-th sub-carrier

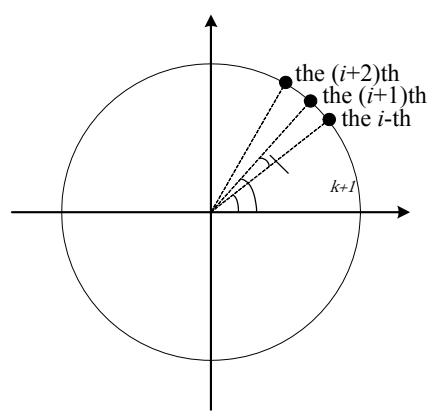

the $(k+1)$ th sub-carrier
Figure 1. demodulated phase deviation on different sub-carriers at different timez

Now we give a proposition to characterize the influence of SFO (denoted by $\Delta F$ hereafter) on the phase of the demodulated output. Provided the length of FFT is $N$ and the rate of SFO is $\lambda$, then the deviation at the k-th subcarrier $\Delta \theta_{k}$ is given by:

$$
\Delta \theta_{\mathrm{k}}=\lambda \times k \times 2 \pi / N(\mathrm{rad})
$$

The proof is as follows:

Let's consider the OFDM modulation at the transmitter. Supposing the sampling frequency at the transmitter is $F_{0}$ and the length of IFFT is $N$, then the $N$ coefficients yielded by IFFT procedure are equally spaced over the frequency range $[0,2 \pi]^{2}$, of which the $k$-th coefficient shall take a phase angle as follows:

$$
\theta_{\mathrm{k}}=2 \pi / N \times k
$$

Deviation on the $k$-th sub-carrier is $\left(F / F_{0}\right) \times k$ when the sampling frequency at the receiver is $F$, so:

\footnotetext{
${ }^{2}$ In fact, only the first coefficients corresponding to positive frequencies are of interest.
}

$$
\begin{aligned}
\theta_{\mathrm{k}}^{\prime} & =2 \pi / N \times\left(F / F_{0}\right) \times k \\
& =2 \pi / N \times\left(1+\left(F-F_{0}\right) / F_{0}\right) \times k \\
& =2 \pi / N \times k+2 \pi / N \times\left(\left(F-F_{0}\right) / F_{0}\right) \times k
\end{aligned}
$$

Phase deviation caused by the mismatch between sampling frequency $F$ and $F_{0}$ is $2 \pi / N \times\left(\left(F-F_{0}\right) / F_{0}\right) \times k$.

We suppose $\lambda=\Delta F / F_{0}=\left(F-F_{0}\right) / F_{0}$, then phase deviation for the two consecutive demodulation of the $\mathrm{k}$-th subcarrier $\Delta \theta_{k}$ is:

$$
\Delta \theta_{\mathrm{k}}=\lambda \times k \times 2 \pi / N(\mathrm{rad})
$$

The proof is complete.

Equation (4) reflects the relationship between SFO and demodulated phase error. In particular, it implies that demodulated phase error on each subcarrier is dependent on its center frequency.

When the receiver couldn't determine $\lambda$ or $\Delta \theta_{\mathrm{k}}$, compensation for the demodulated phase deviation caused by $\mathrm{SFO}$ is impossible.

B. Adaptive sampling frequency tracking and

demodulated phase deviation compensation scheme

based on synchronized symbol information

The proposed sampling frequency offset ratio (SFOR) estimation and demodulated phase error compensation scheme works with frame-based OFDM transmission, and we assume that in each frame transmitted there is a training sequence or frame-synchronization pilot with fixed length and content. As the information born by the training sequence or the frame-synchronization pilot is known a priori to the receiver, the proposed scheme will take advantage of it to

\begin{tabular}{|c|c|c|c|c|c|}
\hline \begin{tabular}{c|c} 
CP & $\begin{array}{c}\text { OFDM } \\
\text { symbol } 1\end{array}$
\end{tabular} & $\overline{\mathrm{CP}}$ & \begin{tabular}{|c|} 
OFDM \\
symbol 2
\end{tabular} & ---------- & $\mathrm{CP}$ & $\begin{array}{c}\text { OFDM } \\
\text { symbol M }\end{array}$ \\
\hline $\begin{array}{l}\text { modulated } \\
\text { symbol } 1\end{array}$ & & $\begin{array}{l}\text { odulated } \\
\text { ymbol } 2\end{array}$ & & & $\begin{array}{l}\text { dulated } \\
\text { mbol M }\end{array}$ \\
\hline
\end{tabular}
estimate the rate of phase deviation and then mitigate the phase noise. Without loss of generality, the OFDM message frame is assumed to take the structure illustrated by Fig.2, in which it can be seen the training sequence or frame-sync pilot begins from the M-th symbol.

Figure 2. the transmission frame of OFDM signals

First we derive the rate of SFO $\lambda$ using the demodulated phase of the received super-frame, then obtain the value of $\Delta \theta_{k}$ from (1) and compensate for phase of other symbols. Figure 3 shows the detailed algorithm flow chart. 


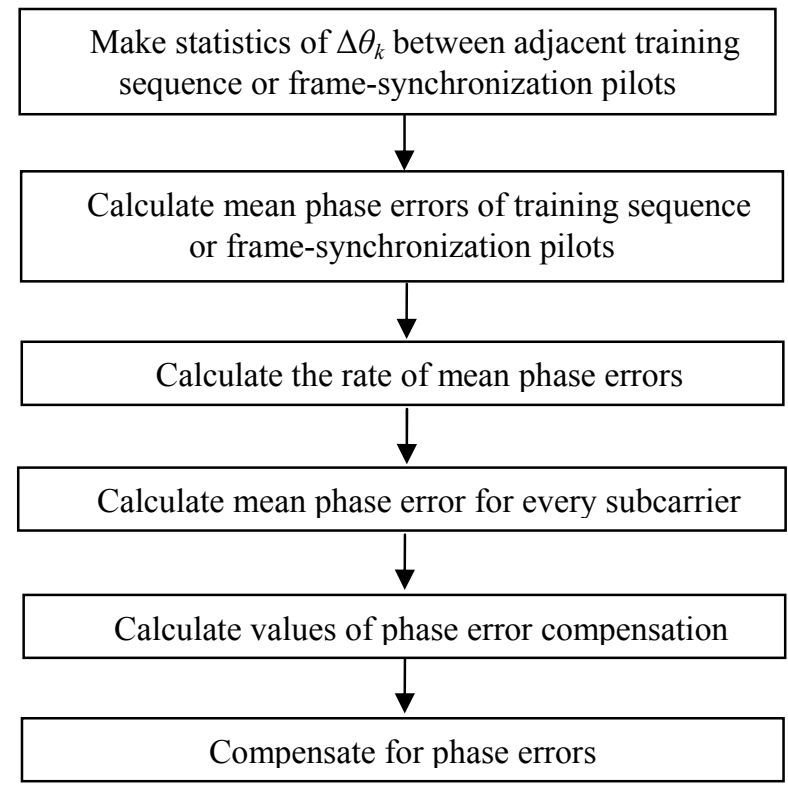

Figure 3. Flow chart of adaptive sampling frequency tracking \& demodulated phase deviation compensation scheme based on synchronized symbol information

After receiving several transmission frames, the receiver demodulates the training sequence or frame-synchronization pilot in every transmission frame and records their phase defined as $\Delta \theta_{1}^{M}, \Delta \theta_{2}^{M}, \ldots, \Delta \theta_{\Delta}^{M}$ for every sub-carrier.

Where:

Subscripts of $\theta$ are index of sub-carriers,

$N$ is the number of sub-carriers,

$M$ indicates that the training sequence or framesynchronization pilot begins from the $M$-th symbol in OFDM transmission frame.

First, we make statistics of the phase difference $\Delta \theta_{1}^{M}, \Delta \theta_{2}^{M}, \ldots, \Delta \theta_{N}^{M}$ between training sequence or framesynchronization pilots for every sub-carrier. Mean phase errors can be expressed as:

$$
\Delta \theta^{M}=\left(\sum_{k=1}^{N} \Delta \theta_{k}^{M} / k\right) / N
$$

Then the rate of phase deviation can be given by:

$$
\lambda=\Delta \theta^{M} / M
$$

Mean phase error on every sub-carrier can be derived by substituting (6) into (7)

$$
\Delta \theta_{K}=\lambda \times K
$$

Where $K$ is equal to $1,2, \ldots, \mathrm{N}$.

Calculate the value of phase error compensation of the $j$ th symbol for the $K$-th sub-carrier in an OFDM transmission frame by substituting (7) into (8):

$$
\Delta \theta_{K}^{j}=\Delta \theta_{K} \times j
$$

Based on the obtained phase error compensation, we shall get $\theta$ of the $j$-th symbol for the $K$-th sub-carrier for every received OFDM transmission frame through demodulation. Then, phase after being compensated can be derived by substituting $\theta$ into (9)

$$
\theta_{K}^{j}=\Delta \theta_{K}^{j}+\theta
$$

Now, $\Delta \theta_{R}^{t}$ is the value of phase after error correction.

III. PERFORMANCE ANALYSIS OF ADAPTIVE SAMPLING FREQUENCY TRACKING \& DEMODULATED PHASE DEVIATION COMPENSATION SCHEME

To evaluate the performance of the proposed adaptive sampling frequency tracking $\&$ demodulated phase deviation compensation scheme, we establish a field test environment based on ADSL signaling. The setting of the test environment is illustrated in Fig.4.

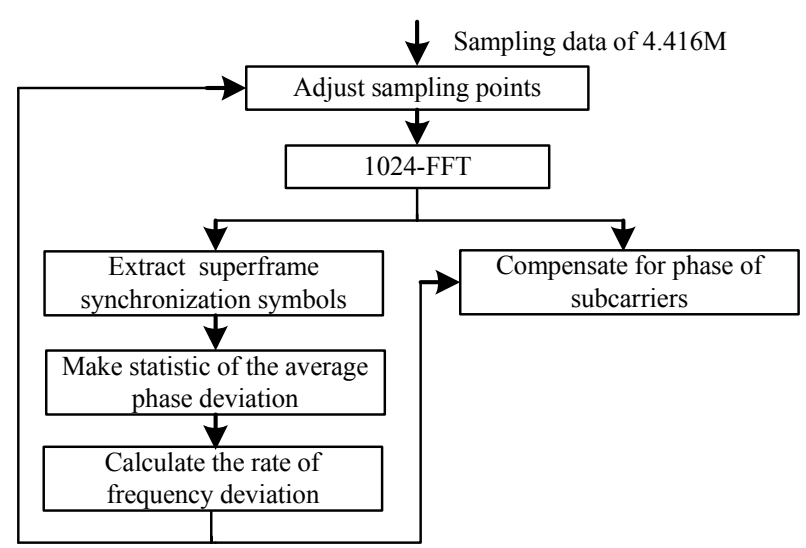

Figure 4. Principle of frequency tracking and phase adjustment

Fig.5 illustrates the field test results for the proposed scheme in the context of a practical ADSL transceiver. Fig.5a is the restored constellation out of synchronization, and Fig. $5 \mathrm{~b}$ is the counterpart when the proposed scheme is equipped. Repeated tests have shown that our SFO estimation and demodulated phase deviation compensation algorithm works well when the SFOR is no larger than $1 \times 10^{\wedge}(-5)$. 


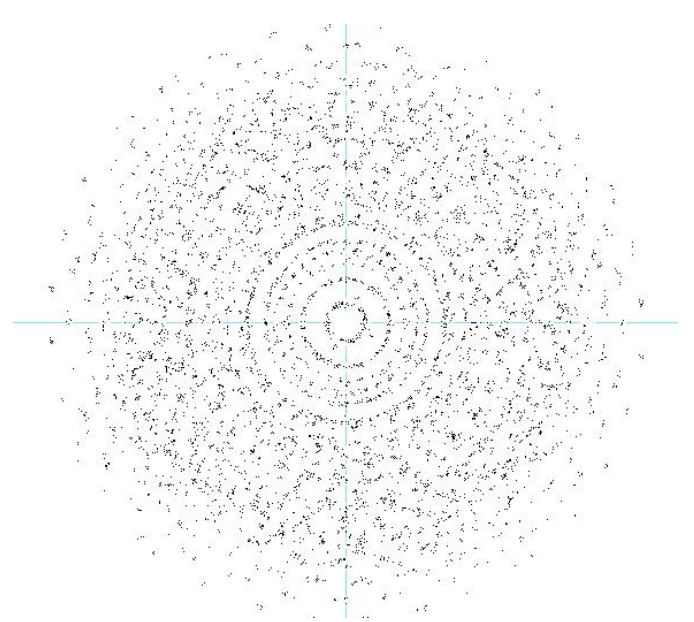

Figure 5a. the restored constellation out of synchronization

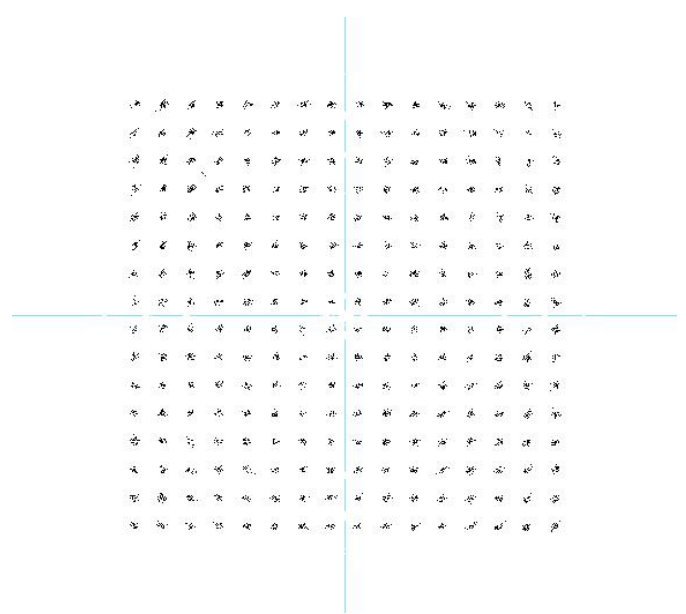

Figure 5b. the restored constellation in synchronization

\section{CONCLUSION}

In this paper, we have studied the influence of SFO between the transmitter \& the receiver on OFDM demodulation. Specially, we have obtained the analytical relationship between the demodulated phase deviation on each subcarrier and its center frequency in the presence of SFO. Following the theoretical analysis, an adaptive pilotbased phase deviation estimation and compensation scheme is proposed, which may greatly improve quality of demodulation for complicated transmitting constellation when SFO exists. Field test results based on a typical ADSL scenario fully testify to the effectiveness of the proposed scheme and its superiority over the receiver where the proposed scheme is not equipped.

\section{REFERENCES}

[1] Hikmet Sari,Georges Karam, and Isa belle Jeandouble, "Transmission techniques for digital terrestrial TV broadcasting," IEEE Comun. Magn.. vol. 33, no.2, pp. 100-109, Feb. 1995.

[2] D.Wood,'European perspectives in digital television Quality, objectives and prospects for commonality," EBU Techno. Rev, no 256,1993,pp,9-15

[3] B. Sueur et al., "The DIGICAST system intended for digital terrestrial television broadcasting," (in French), L'Onde Eledrique, vol. 73, no. 4, July/Aug. 1993, pp. 30-34

[4] R.W.Chang, "Synthesis of band-limited orthogonal signals for multichannel data transmission," Bell Syst. Tech. J., vol. 45, pp. 17751796, Dec. 1966.

[5] Wenbo Wang, Kan Zheng, "Broad wireless communication OFDM technique," Post and Telecom Press, pp. 63-64, Dec. 2003. 